Revista Brasileira de Odontologia Legal - RBOL

\title{
Queiloscopia forense
}

\section{ESTUDO DA ESPESSURA LABIAL COM FINALIDADE PERICIAL - COMPARAÇÃO ENTRE AUTOAVALIAÇÃO E AVALIAÇÃO TÉCNICA*.}

\section{Study of lip thickness for expert purposes - comparison between self- assessment and technical assessment.}

\author{
Brenda Rocha Borba de ANDRADE ${ }^{1}$, Alleson Jamesson da SILVA ${ }^{1}$, Clébia \\ Roberta Eufrazio do NASCIMENTO ${ }^{1}$, Andrezza Ramos dos SANTOS ${ }^{2}$, Adriana \\ Paula de Andrade da Costa e Silva SANTIAGO ${ }^{3}$.
}

1. Cirurgiã(o)-Dentista graduada(o) pela Universidade Federal de Pernambuco, Recife - PE, Brasil.

2. Graduanda em Odontologia pela Universidade Federal de Pernambuco, Recife - PE, Brasil.

3. Professora Adjunta do Departamento de Prótese e Cirurgia Buco-Maxilo-Facial da Universidade Federal de Pernambuco, Recife - PE, Brasil.

*Trabalho de Conclusão do Curso de Odontologia da Universidade Federal de Pernambuco.

\begin{tabular}{ll}
\hline Informação sobre o manuscrito & Autor para contato: \\
Recebido em: 01 Junho 2020 & Profa. Adriana Paula A. da Costa e Silva Santiago. \\
Aceito em: 30 Agosto 2020 & Departamento de Medicina Social - UFPE. \\
& Av. Prof. Moraes Rego, 1235, Campus, Cidade \\
& Universitária, Várzea, Recife, PE, Brasil. CEP. 50740521. \\
& E-mail: adri.odontolegal@gmail.com.
\end{tabular}

\section{RESUMO}

O exame da espessura labial pode se apresentar como uma útil ferramenta orientadora da identificação humana. Neste sentido, este estudo teve como objetivo a avaliação de 200 imagens dos lábios superior e inferior de estudantes da área de saúde da Universidade Federal de Pernambuco, por 02 examinadores, a fim de verificar a concordância entre a avaliação técnica, realizada por eles, e a autoavaliação, realizada pelos examinados, quanto à espessura dos lábios. Para isso, cada examinado inicialmente, após recebimento de instruções e assinatura do TCLE, olhando para um espelho, classificou, sem experiência prévia, seus lábios em finos, médios, grossos ou mistos, segundo sua autoavaliação, o que consideramos como avaliação empírica. Em seguida, o vermelhão dos lábios foi fotografado, associado a uma régua ABFO $\mathrm{n} \div 2$, e mensurado pelos dois examinadores. Os resultados demonstraram que a concordância interexaminadores técnicos, quanto a indicação do tipo de lábios foi alta, se apresentando com um percentual de $98,5 \%$. Em relação à concordância entre a avaliação técnica com a avaliação empírica, foram obtidos valores baixos, ou seja, apresentaram uma concordância pequena, com percentual de $35 \%$. Segundo a avaliação técnica, os lábios grossos se apresentaram com maior porcentagem, 49,5\%, contra 54\% da autoavaliação. $\mathrm{Na}$ autoavaliação, o tipo mais destacado foi lábios médios (54\%). Pode-se concluir que a concordância entre a avaliação técnica e a autoavaliação realizada por pessoa sem experiência (avaliação empírica), neste estudo, quanto ao exame dos tipos de lábio, foi pouco exitosa, em face da pequena coincidência observada, destacando a importância de se considerar seriamente o fato de que tais divergências podem ser altas também em situações onde informações são obtidas a partir de pessoas comuns.

\section{PALAVRAS-CHAVE}

Odontologia legal; Identificação humana; Espessura labial; Fotografia. 


\section{INTRODUÇÃO}

A identificação humana é um processo, muitas vezes, difícil e demorado, e, quando métodos tradicionais odontolegais, o DNA ou mesmo a impressão digital apresentam pouca viabilidade, outros métodos, menos conhecidos, devem ser aplicados para restringir os dados de busca' ${ }^{1}$.

A identificação odontolegal, especificamente, constitui um eficiente método para identificação humana, pois produz resultados plenamente confiáveis e tem como vantagens o baixo custo, facilidade e rapidez na aplicação². Zilio et al. $(2012)^{3}$ elencaram diversos tipos, dentre eles os que utilizam os arcos dentários, a anatomia do crânio, a rugoscopia palatina, fotografia do sorriso, entre outros.

No que se refere aos lábios, a identificação usando suas impressões tem sido descrita na literatura desde a década de 1950, levando a aceitação dessa técnica como evidência para identificação humana, especialmente no sistema judicial, criminal e civil $^{4}$. No entanto, um procedimento que tem encontrado crescente aceitação no campo pericial é a identificação utilizando a fotografia do sorriso. As imagens são empregadas em especial quando os dentes estão expostos, para que sejam comparados com 0 registro a ser examinado ${ }^{3}$.

A fotografia é percebida como uma espécie de prova e espelho do real e ao mesmo tempo é necessária e suficiente para atestar sua veracidade ${ }^{5}$. De acordo com Sontag $(1977)^{6}$ toda fotografia testemunha a dissolução implacável do decorrer do tempo e que as fotografias podem ser mais memoráveis do que imagens em movimento, como por exemplo, filmagens, pois são uma nítida fatia em particular do tempo, e não um fluxo.

Foucault (1999) ${ }^{7}$ já destacava que a fotografia avançou tecnologicamente e isso fez com que ela fosse aplicada em diversas áreas científicas e técnicas e servisse às instituições funcionando como documento/prova e arquivo.

Observa-se, entretanto, que nem sempre as pessoas estão com sorriso aberto nas suas fotografias, o que muitas vezes pode representar uma barreira a identificação odontolegal da mesma. Nestes casos, o exame da espessura labial poderia ser uma útil ferramenta identificatória e sua classificabilidade vem sendo descrita em vários estudos científicos ${ }^{8-13}$.

Diante disso, torna-se importante estudar acerca do emprego da avaliação da espessura dos lábios como meio de orientação da identificação odontolegal. Há diferenças significativas entre a percepção das próprias pessoas acerca da espessura de seus lábios, e o que determina a técnica pericial? Esta questão se põe relevante, visto que, em casos de reconhecimento, a caracterização pessoal, que é empírica, pode ser utilizada como guia, e desta forma, caso a percepção do indivíduo ou testemunha seja muito diferente do que realmente se apresenta, pode ser, de fato um complicador. E, apesar dos diversos trabalhos que se encontram na literatura sobre a espessura labial $^{1,12,14-16}$, nota-se ainda carência bibliográfica de estudos que a discutam como contribuição pericial a identificação odontolegal.

Pelas lacunas de informação sobre da utilização das espessuras labiais como 
coadjuvante para fins periciais, e pela possibilidade da mesma representar um importante primeiro registro para identificação, quando analisadas em fotografias anteriores ao evento investigatório, ou mesmo em situações que requerem um retrato falado, justifica-se a realização deste estudo, e seus desdobramentos, que teve como objetivo analisar o nível de coincidência entre a avaliação da espessura labial, segundo percepção do próprio examinado (autoavaliação) e a avaliação técnica, neste sentido sendo empregada a classificação técnica antropométrica de Santos $(1967)^{8}$.

Santos $(1967)^{8}$ foi considerado um dos pioneiros em classificar os sulcos de lábios, investigando, no Brasil, o emprego das impressões labiais como método de identificação humana.

Após sua investigação, vários foram os trabalhos realizados considerando sua classificação, observando, além da espessura, características dos lábios, disposição das comissuras labiais e impressões deixadas em diversos suportes $^{17-20}$.

\section{MATERIAIS E METÓDO}

O projeto de pesquisa foi aprovado pelo Comitê de Ética em pesquisa da Universidade Federal de Pernambuco UFPE (Parecer consubstanciado do CEP $n$. 3.243.654, Projeto 08541319.2.0000.5208).

Para sua realização, inicialmente cada voluntário respondeu, individualmente, olhando para um espelho, a seguinte pergunta: - qual a espessura dos seus lábios? ( ) finos, ( ) grossos, ( ) médios, ou (
) mistos?. Vale salientar que no Termo de Consentimento Livre e Esclarecido - TCLE, o qual foi antes assinado, havia a definição de cada tipo de lábios, segundo Santos $(1967)^{8}$.

Em seguida, cada um dos examinados teve parte da sua face fotografada (terço inferior), com os lábios em posição de repouso - cerrados, com a presença da régua $A B F O$ n.2, e incluída com um número de registro, em arquivo, no computador do pesquisador responsável pelo referido estudo.

Foram realizadas fotografias coloridas com o paciente sentado com a coluna ereta, utilizando o smartphone iPhone 7 Plus com câmera de 12 megapixels, que reproduz fotos com uma resolução de $4000 \times 3000$ pixels, dos 200 (duzentos) voluntários, independente do gênero, distribuídos igualitariamente entre os dos 10 (dez) cursos da área de Saúde, da Universidade Federal de Pernambuco, o que gerou o número de 20 (vinte) discentes de cada curso, como já referido, escolhidos aleatoriamente.

Os arquivos foram analisados por 02 examinadores, um estudante de odontologia da UFPE do último ano e um especialista em odontologia legal, com finalidade de verificar a concordância dos mesmos, considerando a classificação de Santos $(1967)^{8}$, em grossos, médios, finos ou mistos (Figura 1).

O emprego do smartphone teve como propósito, simular situações do dia a dia das pessoas, visto que tal aparelho é extremamente utilizado para tal finalidade, ou seja, fotografar imagens. 


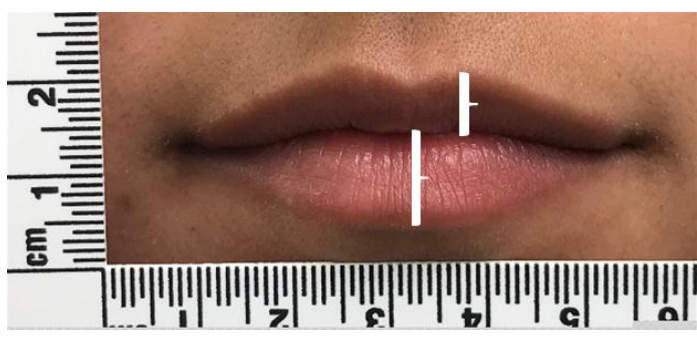

Figura 1. Espessura da zona vermelha ("vermelhões") dos lábios.

Os critérios de inclusão para seleção dos voluntários para o estudo foram ser estudante regularmente matriculado no Centro de Ciências da Saúde, cursando qualquer período, com exceção do curso de Odontologia que deve estar cursando até o $7^{0}$ período, pois neste, é ministrada a disciplina Odontologia Legal, em cujo plano de ensino consta o estudo das técnicas periciais forenses, o que pode influenciar esta pesquisa; maior de 18 anos e que não apresente anomalia nos lábios, que cause maior individualização do seu contorno.

Foram excluídas as avaliações de menores de 18 anos e quem possuísse anomalia nos lábios que impossibilitava sua mensuração.

As análises das imagens, devidamente identificadas e numeradas foram realizadas individualmente pelos examinadores em seu computador pessoal, por meio de uma avaliação cega. Com a finalidade de reduzir a fadiga dos examinadores $e$, consequentemente, de evitar a variabilidade nos resultados das interpretações gráficas, cada examinador foi orientado a realizar repouso entre as avaliações, de acordo com sua necessidade de descanso mental e visual. Todos os examinadores realizaram as avaliações pela manhã, antes de suas atividades diárias, também com o objetivo de padronização.
Foi realizado um estudo com a f na dade de calibração dos examinadores, com 10 imagens de fotografias da região dos lábios, as quais não faziam parte das 200 utilizadas na pesquisa, sendo realizada a avaliação destas por duas vezes, com intervalo de 07 (sete) dias, com intenção de dirimir as possibilidades de diferenças entre as avaliações de ambos. Foi entregue um roteiro impresso a cada examinador, orientando como deveria ser realizada a avaliação das imagens da região do mento até a relativa aos ossos próprios de nariz, conforme se segue:

\section{Avaliação do tipo de lábios:}

- Lábio superior - da linha superior ao

pilar do filtro até a borda inferior do tubérculo do vermelhão labial.

- Lábio inferior - da porção mais alta até a mais baixa do vermelhão do lábio inferior.

\section{E então classificá-lo de acordo} com a sugerida por Santos $(1967)^{8}$ :

- Lábios finos: inferiores a $8 \mathrm{~mm}$;

- Lábios médios: variando de 8 a $10 \mathrm{~mm}$;

- Lábios grossos: superiores a $10 \mathrm{~mm}$; -Lábios mistos: cuja classificação é diferente para o lábio superior e inferior.

Os dados foram analisados descritivamente por meio de frequências absolutas e percentuais. Para avaliar a diferença entre os sexos em relação às avaliações da espessura labial, foi utilizado o teste Qui-quadrado de Pearson ou o teste Exato de Fisher quando a condição para utilização do teste Qui-quadrado não fora verificada $^{21}$. 
Com o objetivo de se determinar o grau de concordância entre examinadores e dos examinadores com os dados da autoavaliação, foram obtidos os escores de concordância de Kappa e intervalos para o referido parâmetro.

A margem de erro utilizada nas decisões dos testes estatísticos foi de $5 \%$ e os intervalos foram obtidos com $95 \%$ de confiança. $O$ programa utilizado para digitação dos dados e obtenção dos cálculos estatísticos foi o IBM-SPSS (v.23).

\section{RESULTADOS}

Dos 200 alunos participantes: 49 $(24,5 \%)$ eram do sexo masculino e 151 $(75,5 \%)$ do sexo feminino.

A Tabela 1 apresenta os resultados da avaliação da espessura labial no grupo total segundo o sexo.

$\mathrm{Na}$ Tabela 2 se apresenta os resultados da concordância entre os dois examinadores e com as autoavaliações.

Tabela 1 - Avaliação da espessura labial no grupo total e segundo o sexo.

\begin{tabular}{|c|c|c|c|c|c|c|c|}
\hline \multirow{2}{*}{$\begin{array}{c}\text { Avaliador } \\
\text { Espessura labial }\end{array}$} & \multicolumn{2}{|c|}{ Masculino } & \multicolumn{2}{|c|}{ Feminino } & \multicolumn{2}{|c|}{ Grupo total } & \multirow[t]{2}{*}{ Valor de $p$} \\
\hline & $\mathbf{N}$ & $\%$ & $\mathbf{N}$ & $\%$ & $\mathbf{N}$ & $\%$ & \\
\hline Total & 49 & 100,0 & 151 & 100,0 & 200 & 100,0 & \\
\hline Examinador 1 & & & & & & & $p^{(1)}=0,546$ \\
\hline Lábios finos & 1 & 2,0 & 1 & 0,7 & 2 & 1,0 & \\
\hline Lábios médios & 4 & 8,2 & 20 & 13,2 & 24 & 12,0 & \\
\hline Lábios grossos & 26 & 53,1 & 73 & 48,3 & 99 & 49,5 & \\
\hline Lábios mistos & 18 & 36,7 & 57 & 37,7 & 75 & 37,5 & \\
\hline Examinador 2 & & & & & & & $p^{(1)}=0,478$ \\
\hline Lábios finos & 1 & 2,0 & 1 & 0,7 & 2 & 1,0 & \\
\hline Lábios médios & 3 & 6,1 & 18 & 11,9 & 21 & 10,5 & \\
\hline Lábios grossos & 26 & 53,1 & 74 & 49,0 & 100 & 50,0 & \\
\hline Lábios mistos & 19 & 38,8 & 58 & 38,4 & 77 & 38,5 & \\
\hline Autoavaliação & & & & & & & $p^{(2)}=0,645$ \\
\hline Lábios finos & 2 & 4,1 & 13 & 8,6 & 15 & 7,5 & \\
\hline Lábios médios & 28 & 57,1 & 80 & 53,0 & 108 & 54,0 & \\
\hline Lábios grossos & 11 & 22,4 & 28 & 18,5 & 39 & 19,5 & \\
\hline Lábios mistos & 8 & 16,3 & 30 & 19,9 & 38 & 19,0 & \\
\hline
\end{tabular}

(1) Através do teste Exato de Fisher

(2) Através do teste Qui-quadrado de Pearson. 
Andrade BRB, Silva AJ, Nascimento CRE, Santos AR, Santiago APACS.

Tabela 2 - Avaliação da concordância entre examinadores e com os resultados do autoavaliador.

\begin{tabular}{lcccc}
\hline Examinadores & \multicolumn{3}{c}{ Concordância } & \multicolumn{2}{c}{ Kappa } \\
& $\mathrm{n}^{(1)}$ & $\%$ & Valor & IC \\
\hline Examinador 1 x Examinador 2 & 197 & 98,5 & 0,975 & 0,950 a 1,000 \\
Examinador 1 x auto avaliação & 73 & 36,5 & 0,172 & 0,101 a 0,243 \\
Examinador 2 x auto avaliação & 70 & 35,0 & 0,158 & 0,089 a 0,227 \\
\hline (1) Através do Escore de Kappa & & & &
\end{tabular}

DISCUSSÃO

$\begin{array}{rcr}\text { Esta } & \text { pesquisa realizou a } \\ \text { comparação entre duas formas de }\end{array}$ observação de espessura dos lábios: uma feita pelo próprio indivíduo, e outra por dois examinadores, os quais empregaram a classificação de Santos $(1967)^{8}$, utilizando para isso a fotografia dos lábios. Tratandose da fotografia como prova, temos diversas afirmações de que a imagem fotográfica tem poder documental ${ }^{23}$. A fotografia é percebida como uma espécie de prova e espelho do real e ao mesmo tempo é necessária e suficiente para atestar sua veracidade $^{5}$. Hoje em dia, com o crescente uso das redes sociais, a popularização das fotografias se tornou ainda maior. E, vale destacar que as redes sociais têm se apresentado como ferramenta de importante valor investigativo quando se trata de pessoas desaparecidas ou também pessoas não identificadas, justificando inclusive a construção de aplicativos forenses para smartphones, como o "Selfie Forensic ID", no qual, utiliza compartilhamento de mídias sociais postadas no instagram, Twitter ou Tumblr, por meio de selfies e outras fotos de rosto ${ }^{24}$.

Foram avaliados 200 discentes da área de saúde da UFPE, que, escolhidos aleatoriamente, estabeleceram uma amostra composta de $49(24,5 \%)$ indivíduos do sexo masculino e 151 (75,5\%) do sexo feminino. A concordância entre os dois examinadores foi considerada ótima, de acordo com a escala sugerida os escores de Kappa. Analisando os resultados inerentes ao nível de coincidência entre as referidas avaliações, foi obtido $98,5 \%$ de concordância inter-examinadores técnicos, totalizando 197 análises, das 200 obtidas e apresentando o valor de kappa de 0,975. Este resultado demonstrou que, independentemente da qualificação dos examinadores, que nesta pesquisa compreendeu um especialista em Odontologia legal e um acadêmico de Odontologia do último ano, quando ambos se encontram treinados, a confiabilidade do método é preservada. Neste sentido, ressalta-se a importância da realização prévia de estudo piloto, que por definição é "um teste, em pequena escala, de procedimentos, materiais e métodos propostos para determinada pesquisa" ${ }^{25}$. Desta forma, corroboraram também com os estudos de Bailer, Tomithche e D'Ely $(2011)^{26}$ no qual enfatizaram ser este "uma miniversão do estudo completo, que envolve a realização de todos os procedimentos 
previstos na metodologia de modo a possibilitar alteração/melhora dos instrumentos na fase que antecede a investigação em si”.

Quando analisados os tipos labiais, e considerados dos dois tipos de avaliação propostos nesta pesquisa, quais sejam a autoavaliação e a avaliação técnica ${ }^{9}$, os resultados demonstraram alta discrepância entre eles. Ou seja, ao serem averiguados os dados obtidos pela comparação interanálises técnicas dos indivíduos que realizaram a autoavaliação, foram encontrados valores de kappa variando de 0,172 a 0,158 , respectivamente para a comparação com o examinador 1 e 2 , o que confere resultados de baixa concordância, com porcentagens variando de $35,0 \%$ a $36,5 \%$, ou seja, 70 e 73 análises concordantes, das 200 obtidas, tais achados podem ser atribuídos a falta de treinamento para análise labial por parte dos entrevistados, uma vez que alguns dos participantes relataram ter 0 hábito de autoavaliar seus lábios em situações anteriores, como ao realizar fotografias e olhar-se ao espelho. Vale salientar, que os voluntários não sofreram influência da escala e autoavaliaram-se de maneira empírica, uma vez que o objetivo deste estudo é comprovar se o reconhecimento pode ter como fator complicador a percepção individual.

Assim, enquanto os resultados apresentados pelo autoexame estabeleceram que, em sua maioria predominavam os lábios do tipo médio, com $54 \%$, para o resultado encontrado pelos examinadores, que avaliaram segundo Santos $(1967)^{8}$ a mensuração utilizando fotografia dos lábios, observando lábios do tipo grossos ou muito grossos em 49,5\% para o examinador 1 e $50 \%$ para 0 examinador 2. Tais resultados não corroboraram com os encontrados pelos estudos de Oliveira, Rabello e Fernandes $(2012)^{19}$, que realizaram a medição da espessura labial em estudantes de graduação do curso de Odontologia, sendo utilizado um compasso de ponta seca e uma régua milimetrada (ABFO n.2). Neste, em relação à espessura labial, $65,4 \%$ do grupo apresentaram lábios mistos, enquanto que $21,2 \%$ do grupo revelaram lábios médios. Os lábios estabelecidos como grosso ou muito grosso apenas foram observados em $9,1 \%$ do grupo, e finos, em 4,8\%. Todavia, tais resultados apenas qualificam determinada população, deste modo, o fato de não se assemelharem a outra não determina qualquer prejuízo a qualquer um dos estudos.

No que refere à variável sexo, quando considerado unicamente a avaliação técnica, houve predominância do tipo labial grosso, para ambos os sexos, apresentando uma porcentagem mais significativa para o sexo masculino, que obteve uma porcentagem de $53,1 \%$, além da concordância perfeita inter-examinadores para este segmento.

Santos et al. $(1967)^{8}$ e Barros et al. $(2006)^{17}$ consideram a espessura labial como mais uma ferramenta útil na identificação forense por permitir durante o estudo queiloscópico, uma visualização inicial das dimensões e conformação existentes nos lábios a serem investigados $^{8,17}$. É valido refletir que as particularidades e características individuais 
de uma pessoa possibilitam sua identificação $^{19}$. Nesse sentido, no momento que for feita uma solicitação para uma vítima ou a um parente, para descrever determinada pessoa, torna-se difícil confiar em sua descrição, para $1^{\circ}$ registro dessas características, em face da diversidade de preconceitos estabelecidos por aquele indivíduo. $\mathrm{Na}$ presente pesquisa, foi encontrada divergência correspondente a $63,5 \%$ entre as autoavaliações e a realizada pelos dois examinadores, por meio de mensuração.

Outro ponto importante, diz respeito as variáveis que podem interferir na percepção, como exemplo, para a construção de um retrato falado. O que difere uma vítima de uma testemunha ocular? O peso emocional, a distância e o receio são fatores que devem ser levados em conta, em todo o processo ${ }^{27}$.

"As mulheres violadas têm tendência a exagerar nos traços do suspeito (...) expressão forte, boca forte (...)"28.

E por fim, é evidente a necessidade de se apresentar um registro inicial ou $1^{\circ}$ registro para que a comparação odontolegal seja realizada. No entanto, não se pode deixar de lembrar que, com as ferramentas manipuladoras de imagens, filtros utilizados cada vez mais frequentemente, e ainda o advento da harmonização facial podem representar barreiras importantes para uma comparação efetiva e segura.

Assim, a certificação de que aquela imagem é original, bem como, a certeza de que após aquela mídia ter sido realizada, o indivíduo não tenha se submetido a alterações plásticas, é requisito obrigatório para se evitar maiores complicações, afinal para ser considerado um bom método de identificação, a característica avaliada deve obedecer a ambos os critérios, biológicos e técnicos. Tecnicamente até pode-se obter algum tipo de controle, visto ser limitado o número de métodos em sua classificabilidade e praticabilidade. Mas ao considerar os critérios biológicos, a possível modificação dos lábios, seja em espessura, cor ou mesmo comprimento, pode ser uma importante barreira a ser vencida.

\section{CONCLUSÃO}

Assim, pode-se concluir que, diante dos valores obtidos, onde foi encontrada divergência correspondente a 63,5\%, quando analisado os 197 lábios concordantes inter-examinadores, é válido refletir acerca do valor e confiabilidade dada a avaliação empírica, mesmo que ainda como mera orientadora para identificação forense, se analisada sob a ótica dos valores indicados pela técnica apresentada por Santos $(1967)^{8}$, destacada nesta pesquisa.

\footnotetext{
ABSTRACT

The examination of lip thickness can be a useful tool for guiding human identification. This study aimed to evaluate 200 (two hundred) images of the upper and lower lips of students in the health area of the Federal University of Pernambuco, by 02 (two) examiners, in order to verify the agreement between the technical evaluation, performed by them, and the self-assessment, performed by the examined, regarding the thickness of the lips. For this, each person examined initially, after receiving the instructions and having signed the TCLE, while looking at a mirror, classified, without previous experience, their lips as thin, medium, thick or mixed, according to their self-assessment, which we consider as empirical evaluation. Then, the vermillion of the lips was photographed, associated with an ABFO ruler $n \div 2$, and measured by the two examiners. The results showed that the inter-examiner technical agreement regarding the indication of the type of lips was high, presenting with a percentage of $98.5 \%$. With regard to the
} 
agreement between the technical evaluation and the empirical evaluation, low values were obtained, that is, they presented a poor agreement, with a percentage of $35 \%$. According to the technical evaluation, thick lips presented a higher percentage, $49.5 \%$, against $54 \%$ of self-assessment. In the self-assessment, the most prominent type was medium lips (54\%). It is concluded that the agreement between the technical assessment and the self-assessment carried out by an inexperienced person (empirical assessment), in this study, regarding the examination of the lip types, was not very successful, due to the poor coincidence observed, highlighting the importance of seriously considering the fact that such divergences can be high also in situations where information is obtained from ordinary people.

\section{KEYWORDS}

Forensic Dentistry; Human Identification; Lip thickness; Photograph.

\section{REFERÊNCIAS}

1. Caldas IM, Magalhaes T, Afonso A. Establishing identity using cheiloscopy and palatoscopy. Forensic Sci Int. 2007; 165: $1-9$.

https://doi.org/10.1016/j.forsciint.2006.04.0 $\underline{10}$

2. Magalhães LV, Dos Santos $P$, Karina $T$, Carvalho KS. O potencial da odontologia legal para a identificação humana das ossadas do departamento médico legal de Vitória/ES. Rev Bras Odontol Leg RBOL. 2015; $2(2): \quad 82-86$. http://dx.doi.org/10.21117/rbol.v2i2.27

3. Zilio F, Basualdo, A, Cruz RA. Meios de identificação odontolegal. VII Mostra de Iniciação Científica e Extensão Comunitária e VI Mostra de Pesquisa de Pós-Graduação. IMED, Passo Fundo: Anais. 2013; p. 1-10.

4. Mohammed RB, Patil RG, Pammi VR, Sandya MP, Kalyan SV, Anitha U. Rugoscopy: Human identification by computer-assisted photographic superimposition technique. J Forensic Dent sci. Rushikonda. 2013; 5(2): 90-95. https://doi.org/10.4103/0975-1475.119771

5. Dubois P. O ato fotográfico e outros ensaios. 12. ed. São Paulo: Campinas; 2009.

6. Sontag S. Sobre fotografia: Ensaios. Companhia das Letras; 1977.

7. Foucault M. Vigiar e punir: nascimento da prisão. Petrópolis: Vozes; 1987.

8. Santos M. Cheiloscopy: a supplementary stomatological means of identification. Brazil Int Microform J Leg Med. 1967; 2: 66.

9. Tsuchihashi Y. Studies on personal identification by means of lip prints. Forensic Sci Int. Lausanne. 1974; 3(3): 233-248.

10. Ustuno H, Kanoh T, Tadokoro O, Inoue K. Preliminary study of post mortem identification using lip prints. Forensic Sci Int. 2005; 149: 129-32. https://www.researchgate.net/deref/http\%3 A\%2F\%2Fdx.doi.org\%2F10.1016\%2Fj.fors ciint.2004.05.013
11. Oliveira JA. Determinação das características queiloscópicas dos graduandos de odontologia da universidade federal da Paraíba. Tese (Mestrado). Universidade Federal da Paraíba; 2010. 1-88p.

12. Pereira C. Medicina Dentária Forense. Lisboa: LIDEL - Edições Técnicas; 2012. p. 63-71.

13. Barros GB. Estudo queiloscópico em estudantes do curso de odontologia da Universidade Estadual de Feira de Santana-BA. Saúde.com. 2016; 2: 1. https://doi.org/10.22481/RSC.V2I1.36

14. Pueyo VM, Garrido BR, Sánchez JAS. Odontología legal y forense. 1 ed. 1994.

15. Sharma P, Saxena S, Rathod V. Cheiloscopy: The study of lip prints in sex identification. J Forensic Dent Sci. 2009; 1(1): 24-7. https://doi.org/10.4103/09742948.50884

16. Saxena S, Sharma P, Gupta N. Experimental studies of forensic odontology to aid in the identification process. J Forensic Dent Sci. 2010; 2(2): 69-76. https://dx.doi.org/10.4103\%2F09751475.81285

17. Barros GB, Silva MD, Galvão, LCC. Estudo queiloscópico em estudantes do curso de odontologia da universidade estadual de feira de Santana - BA. Rev SaúdeCom. 2006; 2(1): 03-11.

18. Reddy LVK. Lip prints: an overview in forensic dentistry. J Advanced Oral Research. 2011; 2(1): 17-20. https://doi.org/10.1177\%2F2229411220110 $\underline{104}$

19. Oliveira JA, Rabello PM, Fernandes LCC. Estudo Queiloscópico em Graduandos de Odontologia. Pes Bras Odontoped Clin Integr. 2012; 12(4): 521-528. https://doi.org/10.4034/PBOCl.2012.124.12

20. Osama $R$, Abdullatif AM, Ismail, AK. Duration of reliability of lip print as physical evidence at scene of a crime. Intl Res $\mathrm{J}$ Appl Basic Sci. 2014; 8: 26-33.

21. Douglas GA. Practical Statistics for Medical Research, Chapman and Hall, Great Britain, London. 1991; p. 611. 
22. Conover WJ. Practical Nonparametric Statistics. 2 ed. New York: John Wiley \& Sons, 1980, p. 495.

23. Valentini C. Fotografia forense: A fotografia como documento e prova na investigação criminal. Monografia. Universidade de Caxias do Sul. Caxias do Sul, RS; 2017. Disponível em: http://www.frispit.com.br/site/wpcontent/uploads/2018/09/CLAUDIAVALENTINI 2017 2FO.pdf

24. Nuzzolese E, Lupariello F, Di Vella G. Selfie identification app as a forensic tool for missing and unidentified persons. J forensic dent sci. 2018; 10 (2): 75. https://doi.org/10.4103/jfo.jfds $80 \quad 17$

25. Mackey A, Gass S. Second language research: methodology and design. Mahwah: Lawrence Erlbaum, 2005. p. 4399.

26. Bailer C, Tomitch LMB, D'ely RCS. Planejamento como processo dinâmico: a importância do estudo piloto para uma pesquisa experimental em linguística aplicada. Rev Intercâmbio. 2011; 24: 129146.

27. Reis MSC. Caraterização do indivíduo: retrato falado e desenhado-retrato robô. Tese (Doutorado). Universidade de Lisboa. Alvalade, Lisboa; 2015. Disponível em: https://repositorio.ul.pt/bitstream/10451/222 47/2/ULFBA TES 860.pdf.

28. Ramos A. Retrato - O Desenho da Presença. Lisboa: Campo da comunicação; 2010. 